MODELING, IDENTIFICATION AND CONTROL, 1987, VOL. 8, NO. 1, 47-50

doi:10.4173/mic.1987.16

\title{
An automated system for incubation of pelagic fish eggs
}

\author{
LEIF J $\emptyset$ RGENSEN†
}

Keywords: Aquaculture, fish, egg incubation, automation

An automated system for incubation of pelagic fish eggs is described. The system has an internal air driven water circulation which separates healthy and dead or strongly infected eggs. A processor controlled, pulsed water exchange provides a strongly reduced water requirement. The equipment has also an automated temperature and salinity control and adjustment.

\section{Introduction}

The cultivation of many economically important species has been greatly helped by the increasing use of artificial fertilization and incubation.

Several egg incubation systems have been designed, most of them used for demersal eggs. Pelagic fish eggs, however, need incubators of another type since the eggs are floating in water of the correct salinity.

During the last half of the nineteenth century several hatcheries for pelagic marine fish eggs were built in Norway, partly based on the success of raising anadromous fish species in USA and the fact that G. O. Sars (1866) had published results on artificial fertilization of cod eggs (Gadus morhua). In the U.S.A. hatcheries for many marine species were built and after a visit there, G. M. Dannevig built a cod hatchery in Flødevigen near Arendal (Norway) in 1882. Another hatchery for plaice (Pleuronectes platessa) was built in Trondheim in 1908. The aim was large scale production of larvae, trying to improve the recruitment of the natural population in the area. The hatching technique of marine fish larvae became safe and most of the hatcheries in USA and U.K. adopted the method developed in Flødevigen. Since Dannevig constructed his apparatus (Dannevig 1910) several egg incubation systems have been designed.

One of the main problems concerning egg incubation is removing dead eggs, which are a substrate for bacteria and fungus and in a few hours will reduce the water quality significantly. Ordinarily these eggs have a higher density than fresh eggs and have a tendency to sink. They are removed once a day, using a siphon.

A system where dead and infected eggs are removed continuously will improve the water quality and reduce the need of bactericides and fungicides. Most hatcheries nowadays use both micro-filtered and UV-sterilized water to prevent bacterial and fungal infections. However, such systems have a strongly restricted water capacity and overloading them leads to reduced effectivity. A better water circulation and a more effective water exchange would thus reduce the water requirement.

The growth of fish embryos depends on temperature (Dannevig 1895, Apstein

Received 15 January 1987.

This paper was presented at the IFAC Symposium on Automation and Dataprocessing in Aquaculture, Trondheim, Norway, 18-20 August 1986.

This paper is reprinted with the permission of IFAC.

+ SINTEF, Division of Applied Chemistry, Group of Aquaculture, N-7034 TrondheimNTH (Norway). 
1909, Ryland and Nichols 1975). In adjusting developing rate and to predict the day of hatching, we also need to control the water temperature in the incubator. Particularly when dealing with some marine species this is of great importance, since acceptable synthetic feed is not yet available, and these larvae have to be offered live feed like ciliates, rotifers or different crustacean larvae. These are again usually fed on marine planktonic algae. Startfeeding of fish larvae thus, at least in most cases, includes many steps that need to be synchronized and a total control is necessary.

The egg density of most marine fishes changes during development (Solemdal 1967), which in several occasions gives some trouble with sinking eggs. In nature these eggs might sink to a deeper water layer reaching water of higher salinity. To prevent such sinking the water salinity might be adjusted during egg development.

We wanted to construct an egg incubator with automatic removement of dead and strongly infected eggs, with a reduced water demand and with individual temperature and salinity control.

\section{Construction}

The incubator (Fig. 1(a)) consists of a cylindrical container with a cone, funnel shaped bottom and a central airlift. The lower part of the funnel is formed as a chamber through which the water is tapped. The chamber works as a trap for dead and strongly infected eggs.
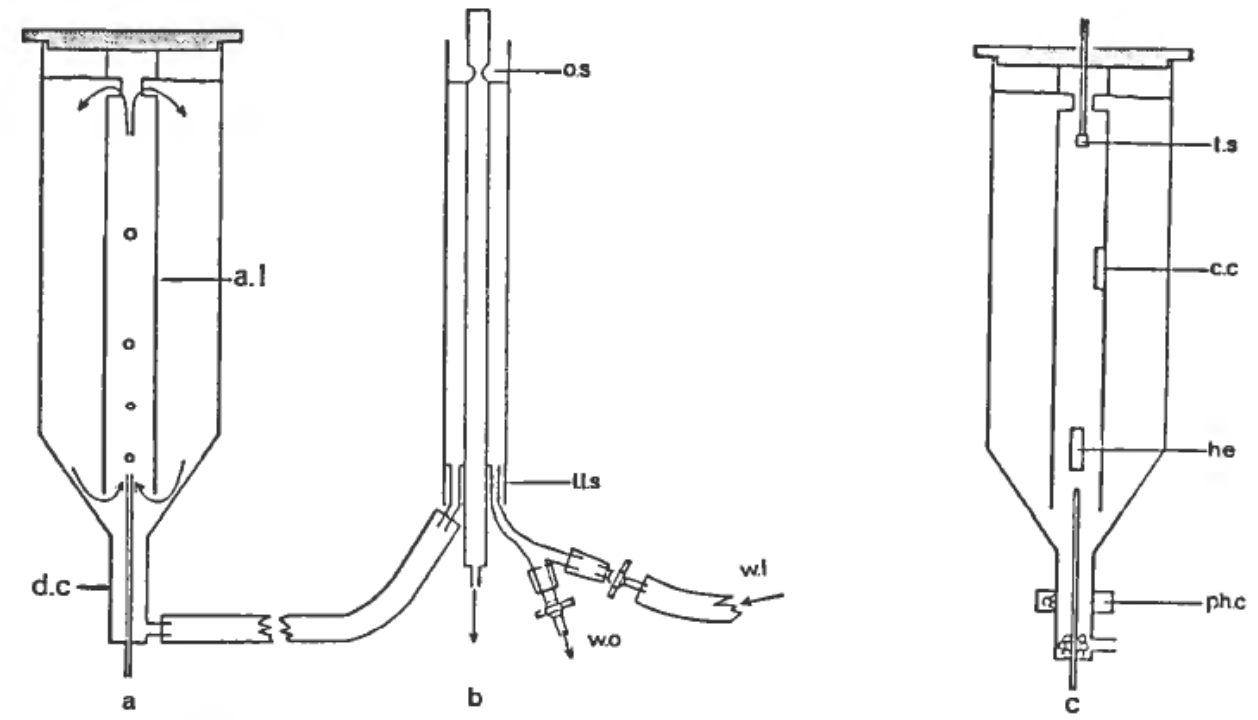

Figure 1. (a) Egg incubator with central air lift (a.l) and dead egg chamber (d.c). (b) External level-column with low-level-siphon (1.1.s); overflow slidge (o.s); water inlet (w.i); water outlet (w.o). (c) Egg incubator with photoelectric cell (ph.c); heating element (h.e); conductivity cell (c.c) and temperature sensor (t.s).

The airlift has two purposes. It produces a careful movement of the eggs preventing dead and infected eggs remaining in the surface film. The other intention is to produce a downward water current along the walls of the funnel preventing dead eggs settling. However, just below the airlift these eggs get into a rut and sink down into the dead egg trap. In this way dead and infected eggs are trapped in a small chamber. The exchange of water between this chamber and the incubator itself is very slow. By introducing pulsed water through the dead egg chamber the eggs are 
removed from the incubator. Usually two different draining procedures are applied; one which involve frequent draining of small amounts of water $(1-5 \%$ of the total volume) to remove the eggs in the chamber, and another more comprehensive draining where about $80 \%$ of the total water volume is replaced.

Since the central air lift creates a nearly homogeneous spreading of the healthy eggs in the water column, some attempts must be made to prevent these eggs being washed out during the draining procedure. By simply stopping the air pump about 5-10 minutes before draining starts the healthy eggs will ascend to the surface. The water is then smoothly tapped until the surface level reaches the upper edge of the funnel. Then new water is let into the incubator.

An external level-column (Fig. 1(b)) provides an exact minimum level as the draining tube allows air through the low-level siphon and the upper level is determined by an overflow slidge. (Another type of level column with two level switches has also been used.) When the correct water level is reached the airpump can commence.

The draining procedures are controlled by a computer (Fig. 2), making it easy to change the time interval between the water exchanges, the delay from stopping the airpump to opening the drain valve and so on (Both magnetic valves and waterpumps have been used). Since one set of level column/level switches, pumps or magnetic valves might be connected to several incubators it is possible to reduce the costs considerably.

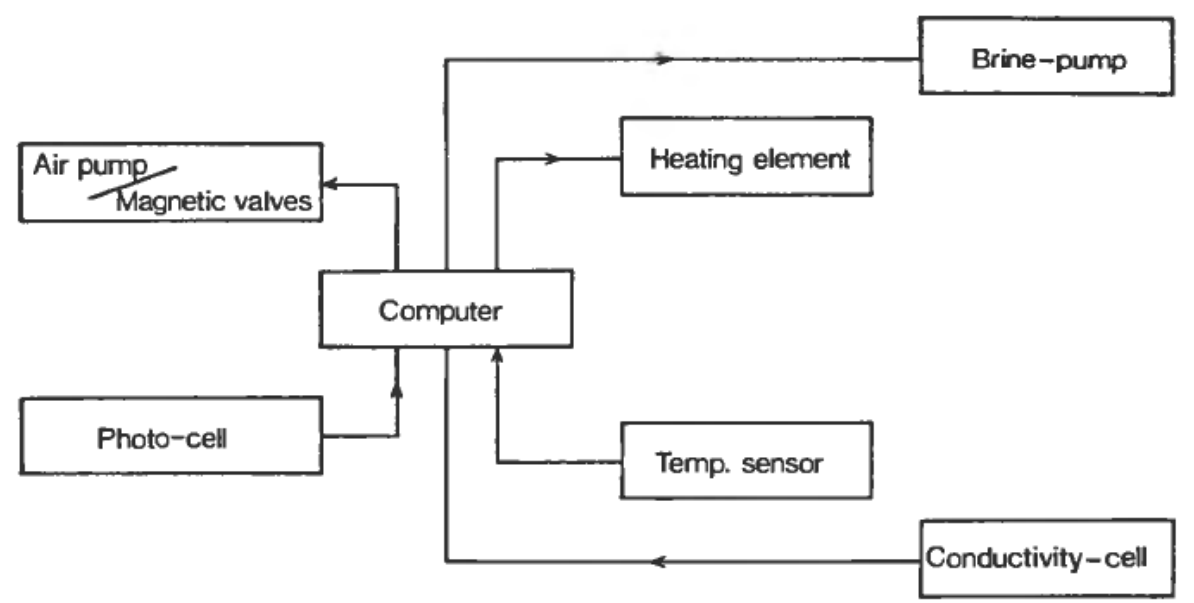

Figure 2. Scheme showing the computer controlled salinity, temperature and automatic removal of dead eggs (triggered by the photo-cell).

A photo-electric cell was mounted to the dead egg chamber (Fig. 1(c)) working in a feedback system to initiate a draining procedure as the amount of dead eggs exceeds a certain limit. An internal, individual, temperature control was built into the system. A temperature sensor (AD-590 with external transmitter) was mounted in the upper part and a small heating element in the lower part of the airlift. The system was controlled by the computer using a PID-controller. The incubator was placed in a refrigerated room and we were able to incubate the eggs at any required temperature above the room temperature. We could also program the computer to follow any temperature gradient if we wanted to simulate natural conditions or to control the rate of embryonic development and thus the day of hatching. The temperature regulated system has been used to study the effect of incubation tem- 
perature on the rate of development and in connection with energy budget measurements of cod and plaice embryos.

The system has also been used with a processor controlled salinity adjustment where any salinity gradient could be followed. A conductivity cell mounted in the airlift was linked to the processor and a pump in a feedback-system. When increasing the salinity the pump was delivering brine, and fresh water when decreasing it. However, when using the automatic, pulsed water-exchange, the salinity adjustment was executed in the reservoir before the water was pumped into the incubator.

\section{Conclusions}

The incubator described has been used during the last six years in hatching plaice, flounder, dab and haddock. Both the water requirement and the cleaning work is significantly reduced compared to traditional incubators. The temperature and salinity control permits adjustment of the hatching time and egg flotation.

\section{ACKNOWLEDGMENTS}

I am greatly indebted to the staff at Biological Station, coworkers at SINTEF, The Scientific Norwegian Council of Fisheries Research (NFFR) and The Royal Norwegian Council of Scientific and Industrial Research (NTNF).

\section{REFERENCES}

APSTEIN, C. (1909). Die Bestimmung des Alters pelagisch lebender Fischeier. Mitt. dt. Seefisch Ver., 25, 364-373.

DANNEviG, G. M. (1895). The influence of temperature on the development of eggs of fishes. Rep. Fishery Bd. Scotl., 1894, 147-152.

DANNEviG, G. M. (1910). Apparatus and methods employed at the marine fish hatchery at Flødevig, Norway. Bull. U.S. Bur. Fish., 28, 801-809.

Ryland, J. S., and Nichols, J. H. (1967). Effect of temperature on the efficiency of growth of plaice prolarvae. Nature, Lond., 214, 529-530.

SARS, G. O. (1866). Om vintertorskens (Gadus morrhua) forplantning og utvikling. Forh. Vid. Selsk. Christiania, 1866.

Solemdal, P. (1967). The effect of salinity on buoyancy, size and development of flounder eggs. Sarsia, 29, 431-442. 